\title{
Complete genome sequence of Pseudomonas citronellolis P3B5, a candidate for microbial phyllo-remediation of hydrocarbon-contaminated sites
}

\author{
Mitja N.P. Remus-Emsermann ${ }^{1 \dagger}$, Michael Schmid ${ }^{2,3+}{ }^{\dagger}$, Maria-Theresia Gekenidis ${ }^{1,4}$, Cosima Pelludat ${ }^{2}$, Jürg E. Frey ${ }^{2}$, \\ Christian H. Ahrens ${ }^{2,3^{*}}$ and David Drissner ${ }^{1 *}$
}

\begin{abstract}
Pseudomonas citronellolis is a Gram negative, motile gammaproteobacterium belonging to the order Pseudomonadales and the family Pseudomonadaceae. We isolated strain P3B5 from the phyllosphere of basil plants (Ocimum basilicum L.). Here we describe the physiology of this microorganism, its full genome sequence, and detailed annotation. The 6.95 Mbp genome contains 6071 predicted protein coding sequences and 96 RNA coding sequences. P. citronellolis has been the subject of many studies including the investigation of long-chain aliphatic compounds and terpene degradation. Plant leaves are covered by long-chain aliphates making up a waxy layer that is associated with the leaf cuticle. In addition, basil leaves are known to contain high amounts of terpenoid substances, hinting to a potential nutrient niche that might be exploited by P. citronellolis. Furthermore, the isolated strain exhibited resistance to several antibiotics. To evaluate the potential of this strain as source of transferable antibiotic resistance genes on raw consumed herbs we therefore investigated if those resistances are encoded on mobile genetic elements. The availability of the genome will be helpful for comparative genomics of the phylogenetically broad pseudomonads, in particular with the sequence of the P. citronellolis type strain PRJDB205 not yet publicly available. The genome is discussed with respect to a phyllosphere related lifestyle, aliphate and terpenoid degradation, and antibiotic resistance.
\end{abstract}

Keywords: Phyllosphere, Complete genome, Pseudomonad, PacBio, Nonhybrid de novo assembly, Alkane degradation, Terpenoid degradation, Bioremediation potential

\section{Introduction}

The genus Pseudomonas encompasses a large group of bacteria that are ecologically and functionally very diverse including many human and plant pathogenic species [1], but also species with mutualistic host interactions, the most prominent examples of which are biocontrol strains that protect plants from pathogens [2-4]. Due to their versatile properties and pathogenicity, pseudomonads garnered

\footnotetext{
*Correspondence: christian.ahrens@agroscope.admin.ch; david.drissner@ agroscope.admin.ch

${ }^{\dagger}$ Equal contributors

${ }^{2}$ Agroscope, Institute for Plant Production Sciences IPS, Wädenswil, Switzerland

${ }^{1}$ Agroscope, Institute for Food Sciences IFS, Wädenswil, Switzerland Full list of author information is available at the end of the article
}

much attention in recent years and members of the genus have been the subject of full genome sequencing projects, i.e. at the time of writing the Pseudomonas Genome Database contained 98 complete genomes and 1447 draft genomes [5]. Up till now, this database is heavily biased towards pathogenic species, most prominently including 996 human-pathogenic $P$. aeruginosa strains and 105 plant pathogenic $P$. syringae strains. Taking into account the assigned species of the sequenced strains only, roughly a third (76 of 199) of the validly published Pseudomonas species [6] have been sequenced. Thus, a comprehensive picture of genetic diversity within the pseudomonads is lacking and investigating thus far unsequenced species will fill this knowledge gap. 
Pseudomonas citronellolis has long been recognized for its ability to degrade hydrocarbons such as isoprenoid compounds including citronellol, hence its name [7], and complex oily sludge contaminations [8], indicating the species might potentially serve as bioremediation organism by cleaning up oil contaminated sites. The described strain was isolated from plant leaf material. Interestingly, plant leaves are covered by a cuticle consisting of cutin, an esterified aliphatic polymer [9] that is impregnated and overlaid by intra- and epi-cuticular waxes consisting of very long-chain aliphatic compounds, including alkanes, alcohols, and fatty acids [10]. The ability of $P$. citronellolis to degrade aliphatic compounds leads to the intriguing possibility that the bacterium may be able to exploit aliphatic compounds as a nutrient source during leaf surface colonization. Furthermore, the ability of $P$. citronellolis to also degrade terpenoid compounds [11] is another interesting feature that might enable this species to degrade the abundantly available terpene compounds from herbal plants [12] and hence may represent a growth advantage over other phyllosphere bacteria on such hosts.

$P$. citronellolis has previously been used as a model organism to investigate metabolic pathways and enzyme activity, such as glucose catabolism and gluconeogenesis [13], acyl-coenzyme A carboxylases [14], and terpene degradation [11]. The complete genome of $P$. citronellolis described here represents a useful resource for the ongoing environmental and physiological research in this species, and can serve as a starting point for detailed systems biology studies [15], at least until the genome sequence of the type strain PRJDB205 becomes available. We present a summary, classification and general physiological features of the strain $P$. citronellolis P3B5, as well as the genomic sequence, assembly, annotation, and its putative adaptations to a phyllosphere lifestyle.

\section{Organism information}

\section{Classification and features}

The species $P$. citronellolis was proposed and described by Seubert [7] and isolated from soil collected under pine trees in northern Virginia, USA. P. citronellolis is a Gram negative, rod-shaped, gammaproteobacterium that is approximately $2 \mu \mathrm{m}$ in length and $0.5 \mu \mathrm{m}$ in width (Fig. 1a), motile by one polar flagellum, and non-sporeforming [7]. On Lysogeny Broth agar P. citronellolis forms white, round colonies (Fig. 1b), that produce green fluorescent pigments after several days of incubation. Grown in liquid M9 minimal medium the production of green fluorescent pigments is even more pronounced (Fig. 1c).

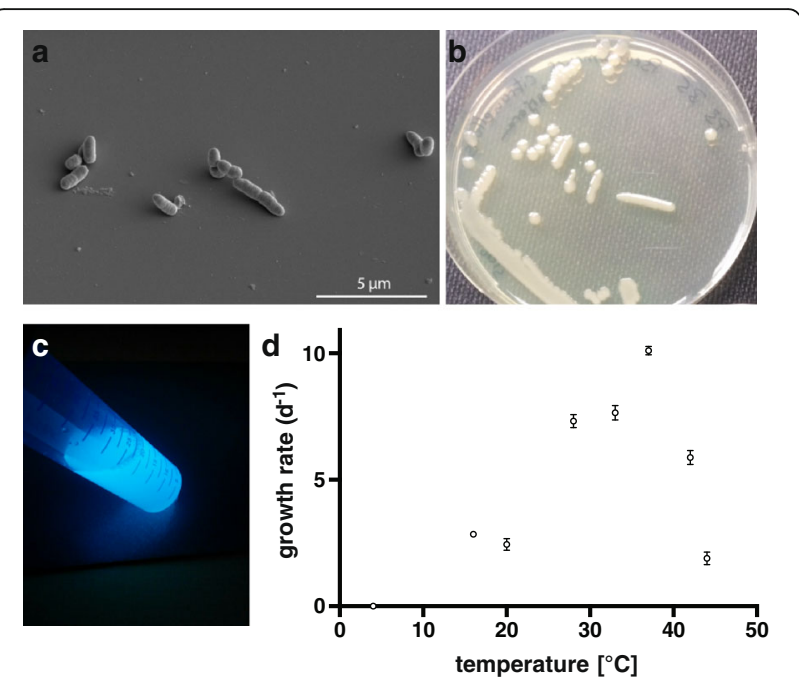

Fig. 1 a Scanning-electron micrograph of $P$. citronellolis P3B5. b $P$. citronellolis P3B5 grown on LB agar for 4 days. $\mathbf{c} P$. citronellolis P3B5 grown in M9 minimal medium for $20 \mathrm{~h}$ excited by UV light exhibiting strong fluorescence. $\mathbf{d}$ Growth of P. citronellolis P3B5 was analyzed by measuring the optical density at $600 \mathrm{~nm}$ at the different temperatures for 24 h. 12 to 15 replicate measurements were performed for each temperature. By plotting the observed growth rate during the exponential growth phase at different temperatures, it was determined that the ideal growth temperature of $P$. citronellolis P3B5 is around $37^{\circ} \mathrm{C}$. No growth was observed at $4{ }^{\circ} \mathrm{C}$

The here-described $P$. citronellolis P3B5 was recovered from healthy basil leaves. The species was initially identified by MALDI biotyping using a MicroFlex MALDITOF mass spectrometer, and the MALDI Biotyper DB V4.0.0.1 (Bruker Daltonics, Germany). MALDI biotyping has been shown to be able to identify pseudomonads at the species level with high accuracy $[16,17]$.

To isolate bacteria from the basil phyllosphere, $30 \mathrm{~g}$ of plant material were suspended in $100 \mathrm{ml}$ peptone water (9 g/L NaCl, $1 \mathrm{~g} / \mathrm{L}$ tryptone (Merck, Germany)), processed for $3 \mathrm{~min}$ in a stomacher (Smasher, AES Chemunex, France) and $100 \mu \mathrm{L}$ aliquots of the resulting supernatant were plated onto different media, including the Escherichia coli selective TBX agar (Oxoid, UK). P. citronellolis P3B5 was isolated from the background microbiota on TBX agar, i.e. non-target bacteria growing on the agar, indicating that $P$. citronellolis utilizes similar compounds as E. coli. Furthermore, since the incubation took place at $44{ }^{\circ} \mathrm{C}$, it is able to grow at high temperatures. However, the characteristic blue color indicative for $E$. coli colonies on TBX agar was not formed by $P$. citronellolis, indicating the absence of a functional $\beta$ glucuronidase in its genome.

During the survey not only P. citronellolis P3B5, but additional, not further characterized $P$. citronellolis isolates were frequently detected in marjoram and basil phyllosphere samples, implying $P$. citronellolis to be part 
of the plants indigenous phyllosphere communities. Pseudomonads are common colonizers of the phyllosphere [18] and can contribute to large proportions of its bacterial community [19-21].

To phylogenetically place $P$. citronellolis P3B5, a phylogenetic tree was constructed by multiple genome alignment using the program progressiveMauve with standard parameters [22] (Fig. 2). From each thus far sequenced species one representative strain, if possible a type strain, was chosen. Only complete genomes were considered. As outgroup species Xanthomonas campestris pv. campestris ATCC 33913 was chosen. A summary of the classification and general features of P. citronellolis P3B5 is given in Table 1.

\section{Biochemical profiling}

To perform a detailed biochemical characterization of $P$. citronellolis P3B5, it was cultivated overnight in $25 \mathrm{~mL}$ MM2 medium containing l-asparagin and sorbitol $\left(4 \mathrm{~g} \mathrm{l}^{-1}\right.$ L-asparagine, $2 \mathrm{~g} \mathrm{l}^{-1} \mathrm{~K}_{2} \mathrm{HPO}_{4}, 0.2 \mathrm{~g} \mathrm{l}^{-1} \mathrm{MgSO}_{4}, 3 \mathrm{~g} \mathrm{l}^{-1}$ $\mathrm{NaCl}, 10 \mathrm{~g} \mathrm{l}^{-1}$ sorbitol) at $28{ }^{\circ} \mathrm{C}$ and $240 \mathrm{rpm}$, before it was harvested by centrifugation at $3500 \times \mathrm{g}$ for $10 \mathrm{~min}$. The harvested cells were washed thrice in $1 \times \mathrm{PBS}$ buffer $\left(8 \mathrm{~g} \mathrm{l}^{-1}\right.$ $\mathrm{NaCl}, 0.2 \mathrm{~g} \mathrm{l}^{-1} \mathrm{KCl}$, 1.44 g l $\mathrm{g}^{-1} \mathrm{Na}_{2} \mathrm{HPO}_{4}, 0.24 \mathrm{~g} \mathrm{l}^{-1} \mathrm{KH}_{2} \mathrm{PO}_{4}$, $\mathrm{pH} 7$ ), before they were resuspended and diluted in $1 \times \mathrm{PBS}$ to reach an optical density of $\mathrm{OD}_{600 \mathrm{~nm}}=0.1$. This suspension was then used for inoculation of Biolog GN2, GenIII, and AN plates (Biolog Inc, USA), which were incubated for several days at $28{ }^{\circ} \mathrm{C}$ and analyzed for changes of their optical density at $590 \mathrm{~nm}$ using a microtiter plate reader (Infinite M200, Tecan, Switzerland).

Analysis of the GN2 plates revealed that $P$. citronellolis P3B5 was able to utilize the following wide range of substrates: 2 -aminoethanol, $\alpha$-ketoglutaric acid, $\alpha$-d-glucose, $\beta$-hydroxy butyric acid, bromo succinic acid, cis-aconitic acid, citric acid, d-alanine, d-cellobiose, d-fructose, $d$ galactonic acid lactone, d-galacturonic acid, d-gluconic acid, d-mannitol, d-mannose, d-melibiose, d-raffinose, d,l- $\alpha$-glycerol phosphate, d,l-lactic acid, $\gamma$-amino butyric acid, glucose-1-phosphate, glucose-6-phosphate, hydroxy-l-proline, inosine, itaconic acid, l-alanine, 1arabinose, l-asparagine, l-aspartic acid, l-glutamic acid, 1-histidine, l-proline, l-pyroglutamic acid, methyl pyruvate, mono-methyl-succinate, p-hydroxy phenylacetic acid,

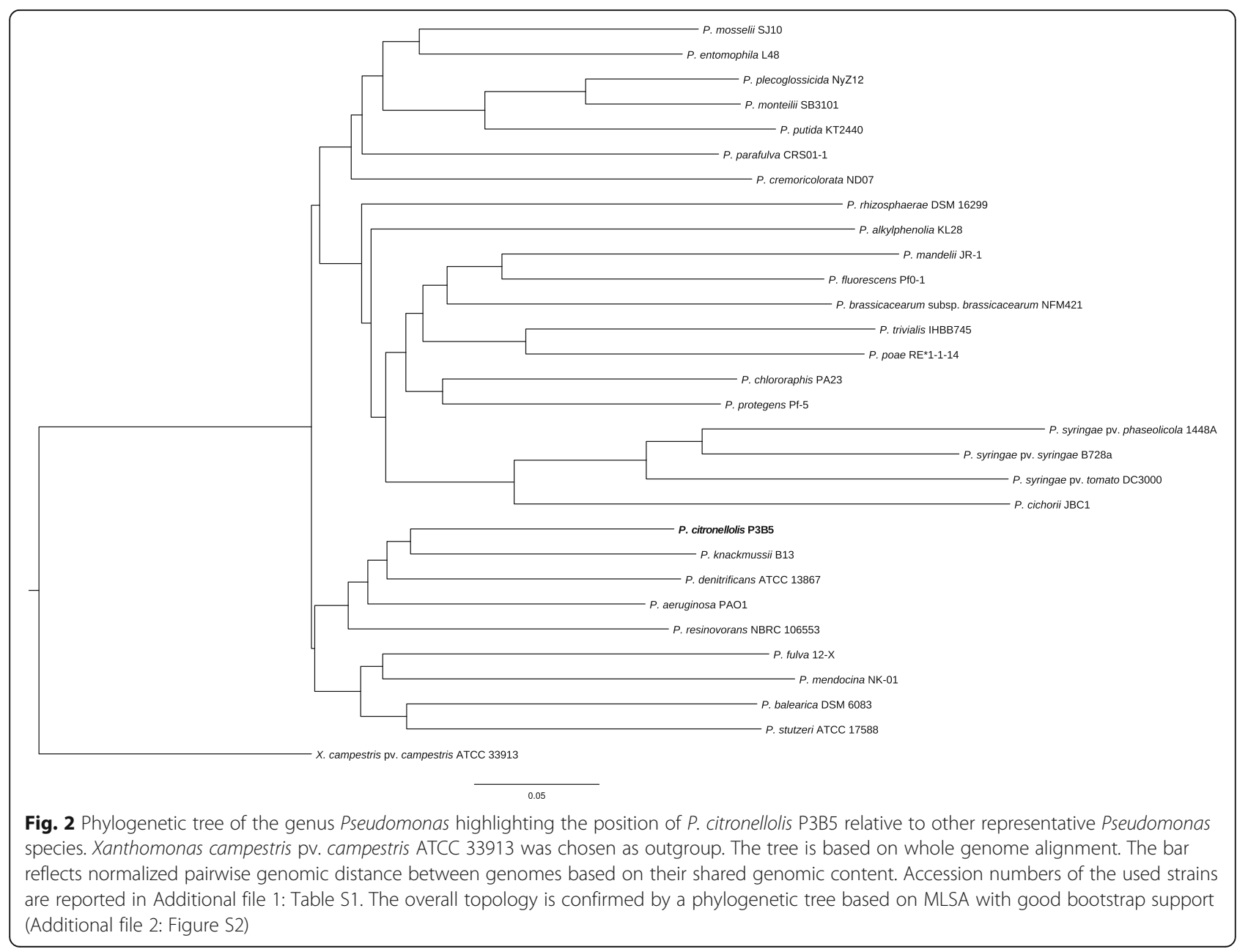


Table 1 Classification and general features of P. citronellolis P3B5 [30]

\begin{tabular}{|c|c|c|c|}
\hline MIGS ID & Property & Term & Evidence code $^{a}$ \\
\hline & Classification & Domain Bacteria & TAS [62] \\
\hline & & Phylum Proteobacteria & TAS [63] \\
\hline & & Class Gammaproteobacteria & TAS [64] \\
\hline & & Order Pseudomonadales & TAS $[65,66]$ \\
\hline & & Family Pseudomonadaceae & TAS $[66,67]$ \\
\hline & & Genus Pseudomonas & TAS $[66,68]$ \\
\hline & & Species Pseudomonas citronellolis & $\operatorname{TAS}[7,23,66]$ \\
\hline & & Type strain LMG 21218 & TAS [7] \\
\hline & Gram stain & Negative & TAS [7] \\
\hline & Cell shape & Rod & TAS [7] \\
\hline & Motility & Motile & TAS [7] \\
\hline & Sporulation & Not reported & TAS [7] \\
\hline & Temperature range & $18-42^{\circ} \mathrm{C}$ & IDA \\
\hline & Optimum temperature & $37^{\circ} \mathrm{C}$ & IDA \\
\hline & pH range; Optimum & not determined & IDA \\
\hline & Carbon source & See paragraph "biochemical profiling" & IDA \\
\hline MIGS-6 & Habitat & Soil, phyllosphere & TAS [7], IDA \\
\hline MIGS-6.3 & Salinity & $1-5 \% \mathrm{NaCl}(\mathrm{w} / \mathrm{v})$ & IDA \\
\hline MIGS-22 & Oxygen requirement & Aerobic & TAS [7] \\
\hline MIGS-15 & Biotic relationship & Free living, symbiont & TAS [7], IDA \\
\hline MIGS-14 & Pathogenicity & Non-pathogen & NAS \\
\hline MIGS-4 & Geographic location & Switzerland/Zurich area & IDA \\
\hline MIGS-5 & Sample collection & 02.07 .2015 & IDA \\
\hline MIGS-4.1 & Latitude & $47^{\circ} 45^{\prime} 37 \mathrm{~N}$ & IDA \\
\hline MIGS-4.2 & Longitude & $8^{\circ} 4^{\prime} 37 \mathrm{E}$ & IDA \\
\hline MIGS-4.4 & Altitude & $521 \mathrm{~m}$ & IDA \\
\hline
\end{tabular}

${ }^{a}$ Evidence codes-IDA Inferred from Direct Assay, TAS Traceable Author Statement (i.e., a direct report exists in the literature), NAS Non-traceable Author Statement (i.e., not directly observed for the living, isolated sample, but based on a generally accepted property for the species, or anecdotal evidence). These evidence codes are from the Gene Ontology project [69]

propionic acid, putrescine, quinic acid, sebacic acid, succinamic acid, succinic acid, sucrose, tween 40, tween 80, and urocanic acid. On Biolog GenIII plates, the following additional substrates were utilized: Acetic acid, $\alpha$-hydroxybutyric acid, $\alpha$-ketobutyric acid, d-fructose-6-phosphate, d-galactose, d-glucose-6-phosphate, d-glucuronic acid, dmalic acid, d-maltose, d-trehalose, formic acid, l-arginine, l-galactonic acid lactone, 1-lactic acid, 1-malic acid, $N$ acetyl-d-neuramic acid, and pectin.

In contrast to previously described $P$. citronellolis strains, P. citronellolis P3B5 could not utilize d-mannitol, glycerol, d,l-carnitine, d-psicose, l-alanyl-glycine, and formic acid. However, compared to the previously described $P$. citronellolis strains, P. citronellolis P3B5 additionally utilized $\alpha-\mathrm{d}-$ lactose, d-galactose, d-glucose-6-phosphate, d-glucuronic acid, d-maltose, d-mannitol, d-melibiose, d-raffinose, dsaccharic acid, d-trehalose, d,l- $\alpha$-glycerol phosphate, glucose-1-phosphate, glucose-6-phosphate, hydroxy-1- proline, inosine, l-arabinose, l-galactonic acid lactone, $\mathrm{N}$-acetyl-d-glucosamine, sucrose, and thymidine [7, 23]. The following compounds, that have not been tested in previous studies, were also utilized by $P$. citronellolis P3B5: $\alpha$-hydroxy-butyric acid, $\alpha$-ketobutyric acid, d-fructose-6phosphate, d-mannose, fumaric acid, l-alanyl-l-histidine, lalanyl-1-glutamine, l-glutamine, 1-lactic acid, 1-pyroglutamic acid, l-rhamnose, l-valine plus 1 -aspartic acid, $N$-acetyl-dneuramic acid, p-hydroxy phenylacetic acid, pyruvic acid, quinic acid, and succinamic acid. In summary, based on the tested set of substrates, $P$. citronellolis P3B5 appears to be metabolically more versatile than previously isolated strains, which might reflect an adaptation to the phyllosphere environment.

Growth of $P$. citronellolis P3B5 at different temperatures $P$. citronellolis was incubated in 12 to 15 replicates each at $44,42,37,33,28,20$, and $16{ }^{\circ} \mathrm{C}$ in tryptic soy broth 
(Oxoid, UK) in a Bioscreen C MBR microwell growth analysis platform (Oy Growth Curves Ab Ltd, Finland). Absorbance at $\mathrm{OD}_{600 \mathrm{~nm}}$ was determined every $30 \mathrm{~min}$ for a total of $24 \mathrm{~h}$ and the specific growth rate of the strain was derived from the exponential growth phase of the cultures. Growth rate at $4{ }^{\circ} \mathrm{C}$ was estimated by incubating a $200 \mathrm{~mL}$ shake flask filled with $50 \mathrm{~mL}$ tryptic soy broth in a cold room for several days and measuring absorbance at $\mathrm{OD}_{600 \mathrm{~nm}}$ after 7 and 9 days using a spectrophotometer (BioPhotometer Plus, Eppendorf, Germany). $P$. citronellolis $\mathrm{P} 3 \mathrm{~B} 5$ is able to grow over a wide range of temperatures, i.e. from 16 to $42^{\circ} \mathrm{C}$, with optimal growth $\sim 37^{\circ} \mathrm{C}$ (Fig. 1d), however, it is unable to grow at $4{ }^{\circ} \mathrm{C}$.

\section{Resilience to abiotic factors and antibiotic resistance}

On Biolog GenIII plates, P. citronellolis P3B5 was able to grow to the same optical density as the positive control in $2 \% \mathrm{NaCl}$ solution, to a lower optical density in $5 \% \mathrm{NaCl}$ solution, and was unable to grow in $9 \% \mathrm{NaCl}$ solution. It was not inhibited by $1 \%$ sodium lactate, rifamycin SV, minocycline, lincomycin, niaproof 4, vancomycin, nalidixic acid, potassium tellurite, and aztreonam. Growth, however not to the same optical density as the positive control, was observed in the presence of fusidic acid, troleandomycin, guanidine $\mathrm{HCl}$, and sodium bromate. No significant growth was detected in presence of $\mathrm{d}$-serine, lithium chloride, and sodium butyrate.

Pseudomonads were previously described to be key players in propagating plasmids, including ABR bearing plasmids, in the phyllosphere [24-28]. Therefore, additional ABR exhibited by P. citronellolis P3B5 were determined in antibiotic disc diffusion assays [29]. Out of the tested 32 clinically relevant antibiotics or antibiotic combinations, $P$. citronellolis P3B5 was resistant against ten antibiotics or combinations including the $\beta$-lactams cefoxitin, cefpodoxime, cefuroxime, temocillin, cephalothin, cefotaxime, the $\beta$-lactam $/ \beta$-lactamase inhibitor mix amoxicillin/ clavulanic acid, and several antibiotics of other classes, i.e. trimethoprim, trimethoprim/sulfamethoxazole, nitrofurantoin, and fosfomycin. P. citronellolis P3B5 was not resistant against the following tested antibiotics: cefepime, colistin, tobramycin, gentamycin, amikacin, ciprofloxacin, levofloxacin, sulfonamide, imipenem, and ceftazidime. Resistance was defined based on existing cutoffs, or, when no cutoff was available, as grown completely up to the antibiotic containing disc [29].

\section{Genome sequencing information Genome project history}

The organism was selected for sequencing as part of an ongoing project investigating the bacterial diversity on the plant surface (i.e., the phyllobiome) of basil (Ocimum basilicum L.). The sequencing project was completed in
December 2015; the sequencing data was deposited as a complete genome (one contig representing the complete genome of P. citronellolis P3B5) in Genbank under BioProject PRJNA309370, with the accession number CP014158. The genome was sequenced with the Pacific Biosciences RS II platform (Microsynth AG, Switzerland). A summary of the project according to the MIGS version $2.0[30]$ is given in Table 2.

\section{Growth conditions and genomic DNA preparation}

To isolate gDNA, cells were grown overnight in terrific broth (12 $\mathrm{g} \mathrm{l}^{-1}$ tryptone, $24 \mathrm{~g} \mathrm{l}^{-1}$ yeast extract, $4 \mathrm{ml} \mathrm{l}^{-1}$ glycerol, $100 \mathrm{ml} \mathrm{l}^{-1} 0.17 \mathrm{M} \mathrm{KH}_{2} \mathrm{PO}_{4}, 0.72 \mathrm{M} \mathrm{K}_{2} \mathrm{HPO}_{4}$ ) at $30{ }^{\circ} \mathrm{C}$ and $250 \mathrm{rpm}$. gDNA was extracted using the GenElute Bacterial DNA Kit (Sigma-Aldrich, USA) according to the manufacturer's recommendations. To concentrate gDNA, the primary eluate was precipitated using $5 \mathrm{M}$ ammonium acetate and $100 \%$ ethanol followed by a washing step using $70 \%$ ethanol. The quality and quantity of the extracted DNA was evaluated on a $1.0 \%(\mathrm{w} / \mathrm{v})$ agarose gel, by measuring absorption ratios $260_{\mathrm{nm}} / 280_{\mathrm{nm}}$ and $260_{\mathrm{nm}} / 230_{\mathrm{nm}}$, and additionally by performing a Qubit dsDNA GR assay (Life Technologies, USA). To determine the presence of plasmids, a plasmid extraction using the NucleoSpin Plasmid kit (Macherey-Nagel, Germany) was performed according to the manufacturer's recommendations. However, no plasmids could be detected after running the sample on a $1.0 \%(\mathrm{w} / \mathrm{v})$ agarose gel.

\section{Genome sequencing and assembly}

The genome of $P$. citronellolis P3B5 was sequenced with one SMRT cell resulting in a total of $1.35 \mathrm{Gbp}$. After quality filtering, 98,808 reads with a mean subread read length of $12,474 \mathrm{bp}$ were obtained (Additional file 2:

Table 2 Project information

\begin{tabular}{lll}
\hline MIGS ID & Property & Term \\
\hline MIGS 31 & Finishing quality & Complete \\
MIGS-28 & Libraries used & $\begin{array}{l}\text { 20 Kb PacBio library (BluePippin } \\
\text { size selection) }\end{array}$ \\
MIGS 29 & Sequencing platforms & PacBio RS II \\
MIGS 31.2 & Fold coverage & 148X \\
MIGS 30 & Assemblers & HGAP.3 \\
MIGS 32 & Gene calling method & Prodigal 2.60 \\
& Locus Tag & PCP3B5 \\
& Genbank ID & CP014158 \\
& GenBank Date of Release & 10.03.2016 \\
& BIOPROJECT & PRJNA309370 \\
MIGS 13 & Source Material Identifier & P3B5 \\
& Project relevance & phyllosphere, environmental, \\
& & biochemistry, and bioremediation \\
\hline
\end{tabular}


Figure S1). Subsequent de novo genome assembly and resequencing steps were performed using PacBio SMRT Portal 2.3.0 [31]. The assembly was generated using the PacBio SMRT Portal protocol RS_HGAP_Assembly.3. Terminal repeats were removed, the genome circularized and its start position was aligned with the dnaA gene using Circlator 1.1.2 [32]. Several rounds of sequence polishing using PacBio SMRT Portal protocol RS_Resequencing.1 with stringent filter criteria ("Minimum Polymerase Read Quality": 84) were performed, resulting in one 6,951,444-bp contig with an average coverage depth of 148 -fold. The assembly did not contain any plasmids and, since virtually all reads were mapped to the genome (97.1\%, other reads were of low quality or chimeric and could still be attributed to the chromosome after manual inspection), provided no evidence for the existence of plasmids. This is in line with the results of the agarose gel electrophoresis analysis described above.

\section{Genome annotation}

Genome annotation was performed using Prokka 1.11.0 [33] with ncRNA search enabled and incorporation of SignalP 4.1 to predict signal peptide cleavage sites [34]. The annotation of selected ORFs annotated as "misc_RNA" by Prokka was manually curated to conform to NCBI requirements. Predicted CDS were then searched against Pfam-A v27.0 [35] and TMHMM 2.0c [36] databases using InterProScan 5.13 [37]. COG categorization was performed by searching predicted CDS against the EggNOG 4.5 database using the Gammaproteobacteria specific dataset [38] and subsequent extraction of COG categories.

\section{Genome properties}

The properties of the complete genome sequence of $P$. citronellolis P3B5 are summarized in Table 3. The average GC content was $67.11 \%$. Of the 6169 predicted genes, 6071 (98.41 \%) were protein CDS of which 4762 genes had a function prediction. Genes without functional prediction by Prokka were annotated as "hypothetical protein". Two pseudo genes (PcP3B5_29180 and PcP3B5_42810) were predicted by the NCBI annotation pipeline [39] and then incorporated into the Prokka annotation. Of the predicted protein coding genes, 5523 were assigned to COGs of 22 classes (Table 4, Fig. 3). Using the Pfam database, 5242 CDS were assigned to a protein family. Putative transmembrane domains were predicted for 1263 CDS. A signal peptide was predicted for 702 CDS. A total of 96 RNA genes were predicted including 15 rRNA (five complete rRNA operons each comprising a 23S, $16 \mathrm{~S}$ and 5S rRNA gene), 76 tRNA, 1 tmRNA, and 4 ncRNA genes. Binding sites for cobalamin and thiamine pyrophosphate were predicted by the NCBI annotation pipeline. One CRISPR repeat was predicted by Prokka and further confirmed by CRISPRFinder
Table 3 Genome statistics

\begin{tabular}{lrr}
\hline Attribute & \multicolumn{1}{c}{ Value } & \% of Total \\
\hline Genome size (bp) & $6,951,444$ & 100.00 \\
DNA coding (bp) & $6,028,113$ & 86.72 \\
DNA G + C (bp) & $4,665,300$ & 67.11 \\
DNA scaffolds & 1 & 100.00 \\
Total genes & 6169 & 100.00 \\
Protein coding genes & 6071 & 98.41 \\
RNA genes & 96 & 1.56 \\
Pseudo genes & 2 & 0.03 \\
Genes in internal clusters & 4762 & NA \\
Genes with function prediction & 5523 & 89.53 \\
Genes assigned to COGs & 5242 & 84.97 \\
Genes with Pfam domains & 702 & 11.38 \\
Genes with signal peptides & 1263 & 20.47 \\
Genes with transmembrane helices & 1 & \\
CRISPR repeats & &
\end{tabular}

[40] and PILER-CR [41]. However, no evidence for a Cas protein-coding gene was found in the genome. An analysis of putative prophages using PHAST [42] resulted in six hits in the genome, three of which were designated as intact prophages (PcP3B5_02970PcP3B5_03410; PcP3B5_06890-PcP3B5_07120; PcP3B5_ 45940-PcP3B5_46520) and the other three as incomplete prophages (PcP3B5_40450-PcP3B5_40640; PcP3B5_408 70-PcP3B5_41140; PcP3B5_46670-PcP3B5_46960). Genomic islands and $A B R$ genes within genomic islands were predicted using IslandViewer 3 [43]. As references for genomic island analysis, the closely related genomes of $P$. knackmussii B13, P. denitrificans ATCC 13867, P. aeruginosa PAO1, and P. stutzeri DSM 4166 were chosen.

\section{Extended insights from the genome sequence \\ The $P$. citronellolis P3B5 genome in the light of a phyllosphere associated lifestyle}

Plant leaf surfaces, often referred to as phyllosphere, represent an extreme environment to its colonizers which are exposed to largely fluctuating levels of drought, DNA-damaging UV radiation, heat, and oligotrophic nutrient conditions [18]. At the micrometer scale the phyllosphere is very heterogeneous, i.e. habitable sites and nutrient availability is discontinuous, tremendously impacting its colonizers $[44,45]$.

\section{Resilience to phyllosphere stress factors}

P. citronellolis P3B5 did not only survive, but grew at temperatures as high as $44{ }^{\circ} \mathrm{C}$ and was also able to grow at moderate temperatures of $16{ }^{\circ} \mathrm{C}$. This ability might represent a fitness advantage on plant leaf surfaces that often feature fluctuating and high temperatures [18]. 
Table 4 Number of genes associated with general COG functional categories

\begin{tabular}{|c|c|c|c|}
\hline Code & Value & $\%$ age & Description \\
\hline J & 189 & 3.11 & Translation, ribosomal structure and biogenesis \\
\hline A & 1 & 0.02 & RNA processing and modification \\
\hline K & 480 & 7.91 & Transcription \\
\hline L & 180 & 2.96 & Replication, recombination and repair \\
\hline B & 5 & 0.08 & Chromatin structure and dynamics \\
\hline D & 40 & 0.66 & Cell cycle control, Cell division, chromosome partitioning \\
\hline V & 75 & 1.24 & Defense mechanisms \\
\hline $\mathrm{T}$ & 286 & 4.71 & Signal transduction mechanisms \\
\hline M & 281 & 4.63 & Cell wall/membrane biogenesis \\
\hline N & 114 & 1.88 & Cell motility \\
\hline U & 79 & 1.30 & Intracellular trafficking and secretion \\
\hline O & 182 & 3.00 & Posttranslational modification, protein turnover, chaperones \\
\hline C & 417 & 6.87 & Energy production and conversion \\
\hline G & 196 & 3.23 & Carbohydrate transport and metabolism \\
\hline$E$ & 489 & 8.05 & Amino acid transport and metabolism \\
\hline $\mathrm{F}$ & 112 & 1.84 & Nucleotide transport and metabolism \\
\hline H & 160 & 2.64 & Coenzyme transport and metabolism \\
\hline । & 218 & 3.59 & Lipid transport and metabolism \\
\hline P & 349 & 5.74 & Inorganic ion transport and metabolism \\
\hline Q & 142 & 2.34 & Secondary metabolites biosynthesis, transport and catabolism \\
\hline $\mathrm{R}$ & 0 & 0.00 & General function prediction only \\
\hline$S$ & 1528 & 25.17 & Function unknown \\
\hline- & 548 & 9.03 & Not in COGs \\
\hline
\end{tabular}

The total is based on the total number of protein coding genes in the genome

Adaptation to fluctuating and high temperatures is also reflected in the genome which encodes several heat shock proteins including DnaK (PcP3B5_54370, PcP3B5_56190), GroEL (PcP3B5_12480), and the cold shock proteins CspA_1-CspA_4 (PcP3B5_06040, PcP3 B5_17140, PcP3B5_45760, PcP3B5_47880). To counter oxidative stress, P. citronellolis $\mathrm{P} 3 \mathrm{~B} 5$ is equipped with genes encoding proteins known to be involved in oxidative stress reduction such as a manganese-based superoxide dismutase (PcP3B5_11610), a ferrousbased superoxide dismutase (PcP3B5_52370), four catalases (PcP3B5_07860, PcP3B5_23220, PcP3B5_27040, PcP3B5_31600), and several peroxidases (PcP3B5 10150, PcP3B5_32470, PcP3B5_44760). To counter drought stress, $P$. citronellolis P3B5 is equipped with genes encoding for the production of trehalose (PcP3B5_27330) and uptake or production of betaine (PcP3B5_00410-00420, PcP3B5_01390, PcP3B5_013 30-01360, PcP3B5_17690, PcP3B5_02060-02090, PcP 3B5 25880, PcP3B5 26750-26770, PcP3B5 28710-28 740, PcP3B5_28870, PcP3B5_29850, PcP3B5_3173031770, PcP3B5_34470-34500, PcP3B5_34590, PcP 3B5_36730, PcP3B5_43400, PcP3B5_45400, PcP3B5_
48640, PcP3B5_50450, PcP3B5_58450-58430, PcP 3B5_60970, PcP3B5_61100, PcP3B5_61120-61140, Pc P3B5_61750, PcP3B5_62040), two osmoprotectants. Notably, no other representative pseudomonad currently found in the SEED database [46] features as many betaine related uptake systems, indicating the importance of this osmoprotectant for the lifestyle of P. citronellolis P3B5. This suggests a phyllosphere adapted lifestyle which requires the ability to survive and thrive under constant drought stress. The phyllosphere has furthermore been described to be iron limited [47], therefore, the acquisition of and competition for iron is crucial on plant leaves. The P. citronellolis P3B5 genome, as is common in pseudomonads, encodes for several genes involved in the acquisition of iron by the production and uptake of siderophores.

\section{Potential resource utilization on plant surfaces}

P. citronellolis P3B5 is equipped for the acquisition and utilization of many nutrients that are available in the phyllosphere, especially different carbohydrates and amino acids [48, 49]. Genes encoding for ABC transporters for amino acids found on plant surfaces 


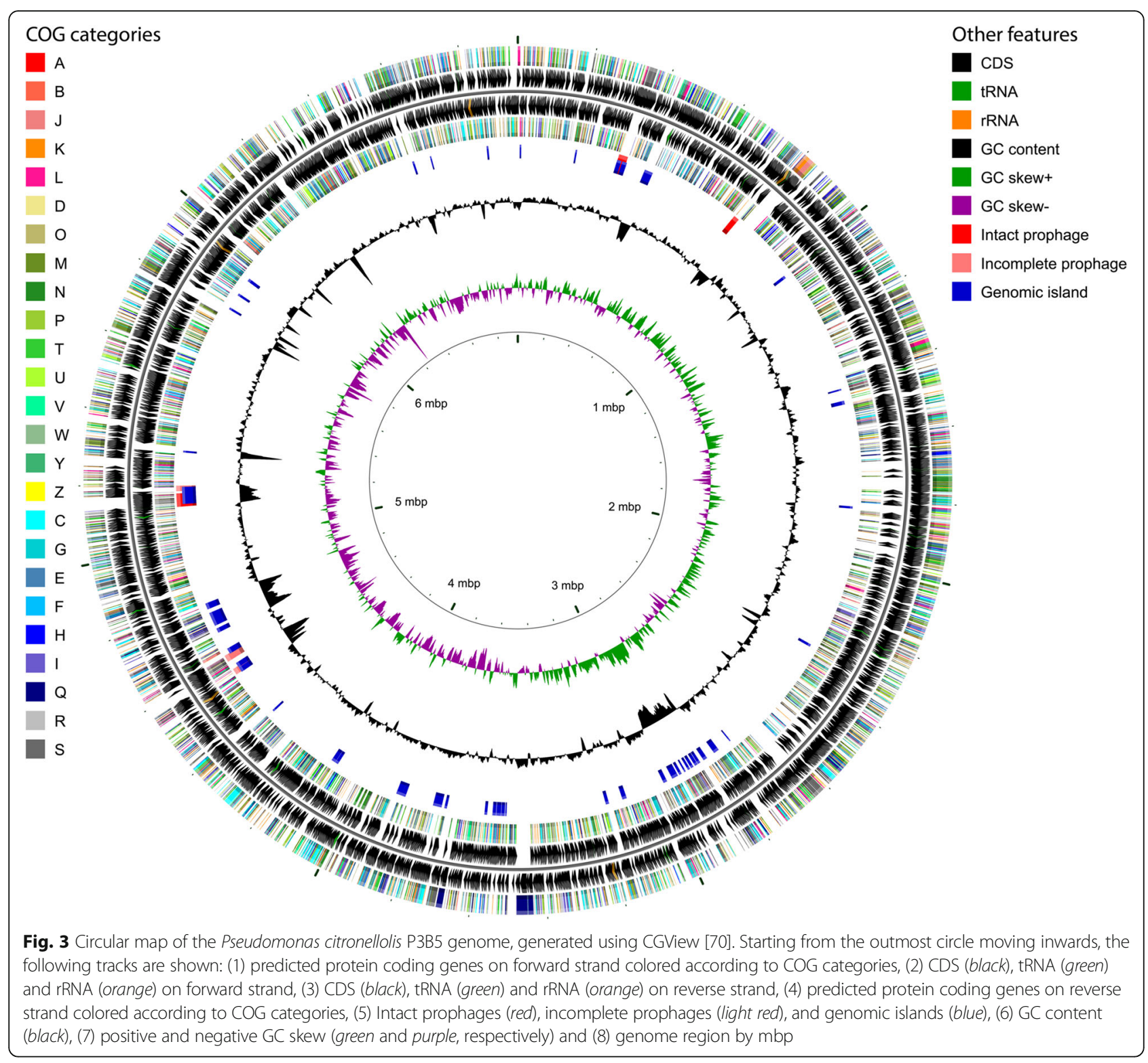

(arginine, cysteine, glycine, histidine, methionine, proline), dipeptides, oligopeptides, branched amino acids, putrescine, spermidine, lipopolysaccharides, glucose, and nitrate. Furthermore, genes encoding for a phosphotransferase uptake system for fructose are present, as well as major facilitator superfamily protein sugar transporter and other sugar transporters. To predict the ability of $P$. citronellolis P3B5 to metabolize various compounds, RAST [50] and modelSEED were employed (http://modelseed.org) [46, 51, 52]. Genetic evidence for the metabolic utilization of glucose, fructose, and sucrose, the three most prevalent photosynthates found on leaves, was found $[48,53]$. Furthermore, genes predicted to be involved in the degradation of short organic acids which can be found on plant leaves were detected, e.g. citrate, fumarate, glycolate, malate, maleate, pyruvate, succinate, and tartrate [48].

Recently, the soil-borne Pseudomonas aeruginosa SJTD-1, a strain phylogenetically related to P. citronellolis, was shown to be able to degrade medium and long chain alkanes from n-tetradecane $\left(\mathrm{C}_{14}\right)$ to $n$-tetracosane $\left(\mathrm{C}_{24}\right)$ due to the protein alkane monooxygenase [54]. The presence of two AlkB-like alkane monooxygenase genes in the $P$. citronellolis P3B5 genome (PcP3B5_23990, PcP3B5_31900) might enable it to nutritionally access long and very-long chain alkanes available on plant leaves, thereby gaining a fitness advantage over other phyllosphere colonizers. Furthermore, P. citronellolis P3B5 contains two putative AlmA-like monooxygenases (PcP3B5_03500, PcP3B5_37240), which would 
allow the degradation of very long chain alkanes $\left(>\mathrm{C}_{30}\right)$ [55]. P. citronellolis P3B5 was isolated from herbs that are rich in aromatic oils, such as terpenes [12]. Previously it has been shown that $P$. citronellolis is able to degrade terpenes such as citronellol [11] and a corresponding gene cluster (PcP3B5_19950-20010) was also detected in P. citronellolis P3B5, which may be responsible for enabling this species to colonize the phyllosphere of aromatic herbs.

\section{Biotic relationship to the plant host}

P. citronellolis P3B5 and other P. citronellolis isolates were isolated from healthy plant material and plants from the same field plot did not exhibit disease symptoms during the course of the survey. Therefore, it is unlikely that $P$. citronellolis is a plant pathogen. It is unclear if the strain is able to confer a health promoting effect on its plant host, thus it is prudent to classify it as tritagonist, an organism whose biotic relationship to its host is thus far undescribed [56]. However, the P. citronellolis $\mathrm{P} 3 \mathrm{~B} 5$ genome contains genes whose products are involved in the biosynthesis of indole-3-acetic acid (PcP3B5_05210-05220, PcP3B5_17810, PcP3B5_0712007140), a compound often found to be produced by bacteria associated with plants [18]. Indole-3-acetic acid is a plant hormone of the auxin class which induces plant cell elongation and division, which leads to an increase of local available nutrients [57]. This indicates that $P$. citronellolis $\mathrm{P} 3 \mathrm{~B} 5$ is able to impact on the plant host.

\section{Antibiotic resistance and possible horizontal gene transfer of resistance genes}

The genomic data broadly supports the phenomenological antibiotic screens described above, the respective putative ABR genes and loci are summarized in Table 5 . Many of the tested compounds were $\beta$-lactam antibiotics of different generations. The P3B5 genome contains genes encoding for six predicted $\beta$-lactamases, providing resistances against many $\beta$-lactam antibiotics, including a class $C \beta$-lactamase conferring resistance to cephalosporin antibiotics and putatively to the monobactam aztreonam. Furthermore, the genome contains genes encoding for efflux pumps that provide resistances to other antibiotics, such as trimethoprim.

To further analyze the potential of described ABR genes to be mobilized by horizontal gene transfer, it was investigated if they are located on mobile genetic elements, i.e. prophages, transposons, and genomic islands. None of the six putative prophage related genomic loci or the predicted genomic islands contained genes encoding for ABR. Furthermore, after close inspection of flanking regions $30 \mathrm{kbp}$ up- and downstream of resistance genes, no evidence for functional transposable elements could be detected.

Table 5 Identified antibiotic resistances and their putative genetic background

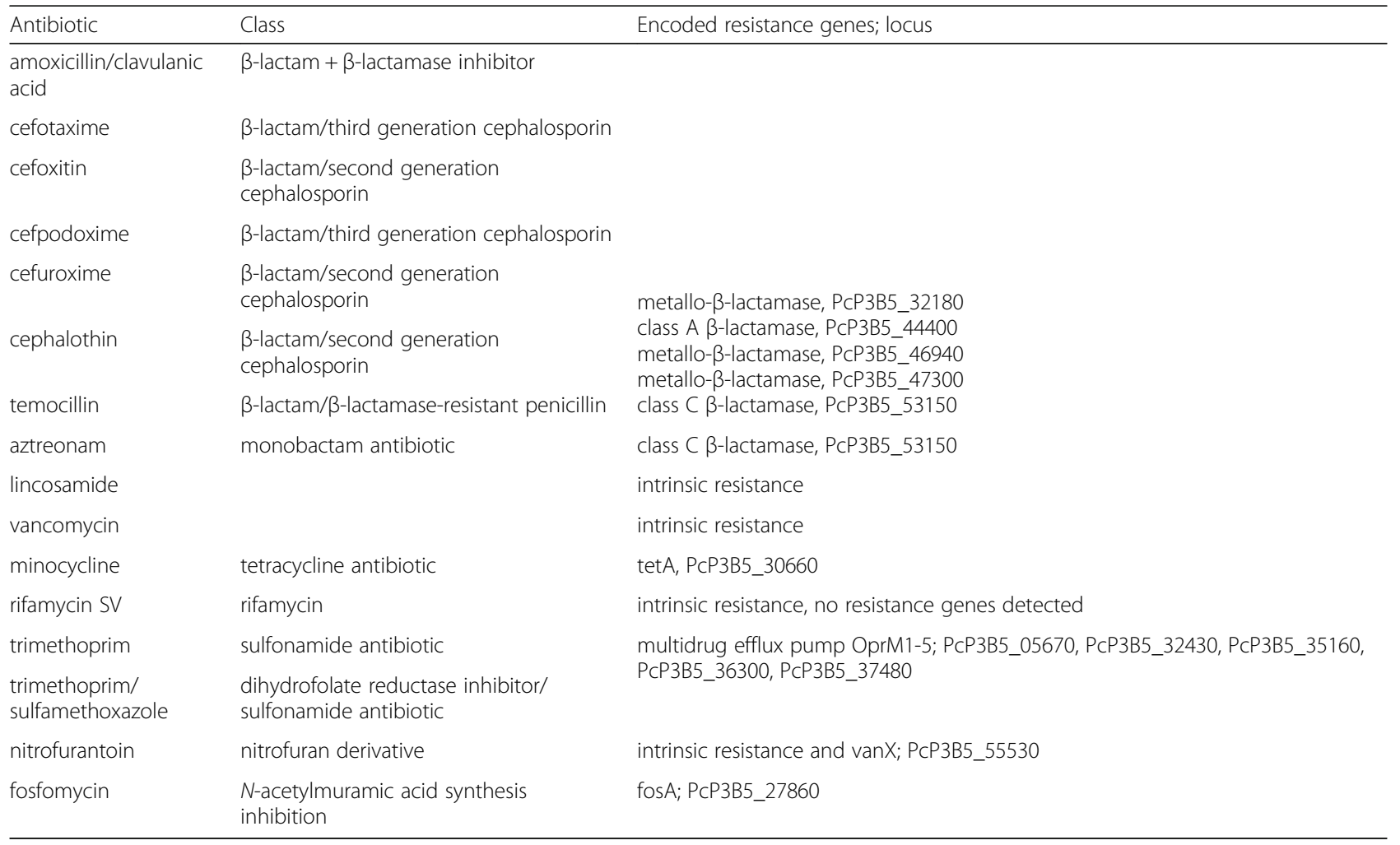




\section{Bioremediation potential}

The capability of $P$. citronellolis P3B5 to degrade longchain alkanes and terpenes in combination with a predicted resilience to many environmental stresses make it an ideal candidate for future bioremediation applications. Also, a recent study highlighted the ability of a $P$. citronellolis isolate to be able to degrade low density polyethylene [58] and, moreover, P. citronellolis P3B5 is closely related to $P$. knackmussi, which is known for its potential in biodegradation of chloroaromatic compounds [59]. This further hints towards a, yet to be further investigated, broad biodegradation potential of $P$. citronellolis. Its preferred niche on plant surfaces can potentially be further exploited for so-called "phyllo-remediation" approaches during which bacteria are employed to degrade organic-pollutants on plant foliage [60, 61].

\section{Conclusions}

We provide a comprehensive insight into the genome and biochemical properties of the environmentally and biotechnologically interesting species $P$. citronellolis. Furthermore, we provide an interpretation of its environmental life-style on plant leaves from a genomic perspective. The $P$. citronellolis P3B5 genome encodes genes that should enable it to degrade long- and very long-chain alkanes, and terpenes. This metabolic capability, in combination with its stress-resilience and phyllosphere lifestyle, makes the organism an intriguing candidate for phyllo-remediation based bioremediation approaches. Resistance to several ABs was observed and several ABR genes were detected, but no evidence for the potential of ABR gene mobilization could be found. The availability of the complete genome sequence of $P$. citronellolis will facilitate future comparative genomics approaches of the phylogenetically broad genus Pseudomonas, which is currently understudied and biased towards pathogenic species. To fully appreciate the genetic diversity of the genus even more species should be complete genome sequenced in the future. This will also enable hypothesis-driven research on the difference between pathogenic and non-pathogenic species.

\section{Additional files}

Additional file 1: Table S1. Accession numbers of representative Pseudomonas genomes used to generate the phylogenetical trees in Fig. 2 and Additional file 2: Figure S2. (DOCX $70 \mathrm{~kb}$ )

Additional file 2: Figure S1. Subread length distribution after sequencing Figure S2. Phylogenetic tree of the genus Pseudomonas highlighting the position of $P$. citronellolis P3B5 relative to other representative Pseudomonas species. Xanthomonas campestris pv. campestris ATCC 33913 was chosen as outgroup. The tree is based on a MLSA using four housekeeping gene sequences (16S rRNA, gyrB, rpoB, rpoD). The bar represents the number of base substitutions per site. The percentage of replicate trees in which associated taxa clustered in the bootstrap test with 1000 replicates are shown next to the respective branches. Accession numbers of the used strains are reported in Additional file 1: Table S1. (DOCX 295 kb)

\section{Abbreviations}

ABR: Antibiotic resistance; gDNA: genomic DNA; MALDI: Matrix-assisted laser desorption/ionization; MALDI-TOF: MALDI-time of flight; MLSA: Multilocus sequence analysis; TBX agar: Tryptone Bile X-Glucuronide agar

\section{Acknowledgments}

The authors thank Diane Gossin for help with the isolation of the strain and Adithi Ravikumar Varadarajan for constructive discussions on genome annotation and phylogenetic analyses. The authors acknowledge support of the Scientific Center for Optical and Electron Microscopy ScopeM of the Swiss Federal Institute of Technology ETHZ and thank Anne Greet Bittermann (ScopeM) for her excellent help with electron microscopy. Reinhard Zbinden is acknowledged for his help and supervision during antibiotic resistance assays. Ulrich Zürcher is acknowledged for the management of the research program REDYMO. This work was financially supported by the Agroscope research programs REDYMO and MicBioDiv.

\section{Author contributions}

MRE conceived and planned the experimental work, performed the biochemical and growth characterization, isolated DNA, analyzed data and the genome, and wrote the manuscript. MS planned and performed the bioinformatics analysis, assembled the genome, annotated the genome, performed phylogenetic analysis, analyzed data, and contributed to the writing of the manuscript. M-TG performed and analyzed the antibiotic resistance screen. CP performed and analyzed the biochemical and growth characterization. JEF planned the bioinformatics analysis. CHA planned and supervised the bioinformatics analysis, annotated the genome, and wrote the manuscript. DD conceived the study and wrote the manuscript. All authors critically read and approved the final manuscript.

\section{Competing interests}

The authors declare that they have no competing interests.

\section{Author details}

${ }^{1}$ Agroscope, Institute for Food Sciences IFS, Wädenswil, Switzerland. ${ }^{2}$ Agroscope, Institute for Plant Production Sciences IPS, Wädenswil, Switzerland. ${ }^{3}$ Swiss Institute of Bioinformatics, Wädenswil, Switzerland. ${ }^{4}$ ETH Zurich, Institute of Food, Nutrition and Health, Zurich, Switzerland.

Received: 12 March 2016 Accepted: 31 August 2016

Published online: 26 September 2016

\section{References}

1. Peix A, Ramírez-Bahena M-H, Velázquez E. Historical evolution and current status of the taxonomy of genus Pseudomonas. Infect Genet Evol. 2009;9:1132-47.

2. Loper JE, Kobayashi DY, Paulsen IT. The Genomic Sequence of Pseudomonas fluorescens Pf-5: Insights Into Biological Control. Phytopathology. 2007;97:233-8.

3. DeVrieze M, Pandey P, Bucheli TD, Varadarajan AR, Ahrens CH, Weisskopf L, Bailly A. Volatile organic compounds from native potato-associated Pseudomonas as potential anti-oomycete agents. Front Microbiol. 2015; 6:1295.

4. Ramette A, Frapolli M, Saux MF-L, Gruffaz C, Meyer J-M, Défago G, Sutra L, Moënne-Loccoz Y. Pseudomonas protegens sp. nov., widespread plant-protecting bacteria producing the biocontrol compounds 2,4diacetylphloroglucinol and pyoluteorin. Syst Appl Microbiol. 2011;34: 180-8.

5. Winsor GL, Van Rossum T, Lo R, Khaira B, Whiteside MD, Hancock RE, Brinkman FS. Pseudomonas Genome Database: facilitating user-friendly, comprehensive comparisons of microbial genomes. Nucleic Acids Res. 2009;37:D483-8.

6. Taxon Abstract for the genus Pseudomonas. NamesforLife, LLC. http://doi.org/ 10.1601/tx.2552. Accessed 28 Aug 2016.

7. Seubert W. DEGRADATION OF ISOPRENOID COMPOUNDS BY MICROORGANISMS I., Pseudomonas citronellolis n. sp: Isolation and Characterization of an Isoprenoid-Degrading Bacterium. J Bacteriol. 1960;79:426-34. 
8. Bhattacharya D, Sarma PM, Krishnan S, Mishra S, Lal B. Evaluation of Genetic Diversity among Pseudomonas citronellolis Strains Isolated from Oily Sludge-Contaminated Sites. Appl Environ Microbiol. 2003;69:1435-41.

9. Kolattukudy PE. Bio-Polyester Membranes of Plants-Cutin and Suberin. Science. 1980;208:990-1000

10. Jetter R, Schäffer S. Chemical composition of the Prunus laurocerasus leaf surface. Dynamic changes of the epicuticular wax film during leaf development. Plant Physiol. 2001;126:1725-37.

11. Forster-Fromme K, Jendrossek D. Identification and characterization of the acyclic terpene utilization gene cluster of Pseudomonas citronellolis. FEMS Microbiol Lett. 2006;264:220-5.

12. Viña A, Murillo E. Essential oil composition from twelve varieties of basil (Ocimum spp.) grown in Colombia. J Braz Chem Soc. 2003;14:744-9.

13. O'Brien R. Enzymatic analysis of the pathways of glucose catabolism and gluconeogenesis in Pseudomonas citronellolis. Arch Microbiol. 1975;103:71-6.

14. Hector ML, Fall RR. Multiple acyl-coenzyme A carboxylases in Pseudomonas citronellolis. Biochemistry. 1976;15:3465-72.

15. Omasits U, Quebatte M, Stekhoven DJ, Fortes C, Roschitzki B, Robinson MD, Dehio $\mathrm{C}$, Ahrens $\mathrm{CH}$. Directed shotgun proteomics guided by saturated RNA-seq identifies a complete expressed prokaryotic proteome. Genome Res. 2013;23:1916-27.

16. Degand N, Carbonnelle E, Dauphin B, Beretti J-L, Le Bourgeois M, SermetGaudelus I, Segonds C, Berche P, Nassif X, Ferroni A. Matrix-Assisted Laser Desorption lonization-Time of Flight Mass Spectrometry for Identification of Nonfermenting Gram-Negative Bacilli Isolated from Cystic Fibrosis Patients. J Clin Microbiol. 2008;46:3361-7.

17. Dieckmann R, Graeber I, Kaesler I, Szewzyk U, Von Döhren H. Rapid screening and dereplication of bacterial isolates from marine sponges of the Sula Ridge by Intact-Cell-MALDI-TOF mass spectrometry (ICM-MS). Appl Microbiol Biotechnol. 2005:67:539-48.

18. Vorholt JA. Microbial life in the phyllosphere. Nat Rev Microbiol. 2012;10:828-40.

19. Finkel OM, Burch AY, Lindow SE, Post AF, Belkin S. Geographical location determines the population structure in phyllosphere microbial communities of a salt-excreting desert tree. Appl Environ Microbiol. 2011;77:7647-55.

20. Rastogi G, Sbodio A, Tech JJ, Suslow TV, Coaker GL, Leveau JH. Leaf microbiota in an agroecosystem: spatiotemporal variation in bacterial community composition on field-grown lettuce. ISME J. 2012;6(10):1812-22.

21. Yang $\mathrm{CH}$, Crowley DE, Borneman J, Keen NT. Microbial phyllosphere populations are more complex than previously realized. Proc Natl Acad Sci U S A. 2001;98:3889-94.

22. Darling AE, Mau B, Perna NT. ProgressiveMauve: Multiple Genome Alignment with Gene Gain, Loss and Rearrangement. PLoS ONE. 2010;5:e11147.

23. Lang E, Griese B, Spröer C, Schumann P, Steffen M, Verbarg S. Characterization of 'Pseudomonas azelaica' DSM 9128, leading to emended descriptions of Pseudomonas citronellolis Seubert 1960 (Approved Lists 1980) and Pseudomonas nitroreducens lizuka and Komagata 1964 (Approved Lists 1980), including Pseudomonas multiresinivorans as its later heterotypic synonym. Int J Syst Evol Microbiol. 2007;57:878-82.

24. Lilley AK, Bailey MJ, Barr M, Kilshaw K, Timms-Wilson TM, Day MJ, Norris SJ, Jones TH, Godfray HCJ. Population dynamics and gene transfer in genetically modified bacteria in a model microcosm. Mol Ecol. 2003;12:3097-107.

25. Kobayashi N, Bailey MJ. Plasmids isolated from the sugar beet phyllosphere show little or no homology to molecular probes currently available for plasmid typing. Microbiology. 1994;140:289-96.

26. Powell BJ, Purdy KJ, Thompson IP, Bailey MJ. Demonstration of tra + plasmid activity in bacteria indigenous to the phyllosphere of sugar beet; gene transfer to a recombinant pseudomonad. FEMS Microbiol Ecol. 1993;12:195-206.

27. Björklöf K, Nurmiaho-Lassila EL, Klinger N, Haahtela K, Romantschuk M. Colonization strategies and conjugal gene transfer of inoculated Pseudomonas syringae on the leaf surface. J Appl Microbiol. 2000;89:423-32.

28. Normander B, Christensen BB, Molin S, Kroer N. Effect of bacterial distribution and activity on conjugal gene transfer on the phylloplane of the bush bean (Phaseolus vulgaris). Appl Environ Microbiol. 1998;64:1902-9.

29. Jorgensen JH, Turnidge JD. Susceptibility test methods: Dilution and disk diffusion methods. In: Manual of Clinical Microbiology. 11th ed. Washington, DC: American Society of Microbiology; 2015.

30. Field D, Garrity G, Gray T, Morrison N, Selengut J, Sterk P, Tatusova T, Thomson $\mathrm{N}$, Allen MJ, Angiuoli SV, et al. The minimum information about a genome sequence (MIGS) specification. Nat Biotechnol. 2008;26:541-7.

31. Chin C-S, Alexander DH, Marks P, Klammer AA, Drake J, Heiner C, Clum A, Copeland A, Huddleston J, Eichler EE, et al. Nonhybrid, finished microbial genome assemblies from long-read SMRT sequencing data. Nat Methods. 2013;10:563-9.

32. Hunt M, Silva N, Otto T, Parkhill J, Keane J, Harris S. Circlator: automated circularization of genome assemblies using long sequencing reads. Genome Biol. 2015;16:294.

33. Seemann T. Prokka: rapid prokaryotic genome annotation. Bioinformatics. 2014;30(14):2068-9.

34. Petersen TN, Brunak S, von Heijne G, Nielsen H. SignalP 4.0: discriminating signal peptides from transmembrane regions. Nat Methods. 2011;8:785-6.

35. Finn RD, Bateman A, Clements J, Coggill P, Eberhardt RY, Eddy SR, Heger A, Hetherington K, Holm L, Mistry J, et al. Pfam: the protein families database. Nucleic Acids Res. 2014;42:D222-30.

36. Krogh A, Larsson B, Von Heijne G, Sonnhammer EL. Predicting transmembrane protein topology with a hidden Markov model: application to complete genomes. J Mol Biol. 2001;305:567-80.

37. Jones P, Binns D, Chang H-Y, Fraser M, Li W, McAnulla C, McWilliam H, Maslen J, Mitchell A, Nuka G, et al. InterProScan 5: genome-scale protein function classification. Bioinformatics. 2014;30(9):1236-40.

38. Huerta-Cepas J, Szklarczyk D, Forslund K, Cook H, Heller D, Walter MC, Rattei T, Mende DR, Sunagawa S, Kuhn M. eggNOG 4.5: a hierarchical orthology framework with improved functional annotations for eukaryotic, prokaryotic and viral sequences. Nucleic Acids Res. 2015. doi: 10.1093/nar/gkv1248.

39. Tatusova T, DiCuccio M, Badretdin A, Chetvernin V, Ciufo S, Li W. Prokaryotic genome annotation pipeline. 2013.

40. Grissa I, Vergnaud G, Pourcel C. CRISPRFinder: a web tool to identify clustered regularly interspaced short palindromic repeats. Nucleic Acids Res. 2007;35: W52-7.

41. Edgar RC. PILER-CR: fast and accurate identification of CRISPR repeats. BMC Bioinf. 2007;8:1.

42. Zhou Y, Liang Y, Lynch KH, Dennis JJ, Wishart DS. PHAST: A Fast Phage Search Tool. Nucleic Acids Res. 2011;39(Web Server issue):W347-52.

43. Dhillon BK, Laird MR, Shay JA, Winsor GL, Lo R, Nizam F, Pereira SK, Waglechner N, McArthur AG, Langille MGl, Brinkman FSL. IslandViewer 3: more flexible, interactive genomic island discovery, visualization and analysis. Nucleic Acids Res. 2015;43:W104-8.

44. Remus-Emsermann MNP, Tecon R, Kowalchuk GA, Leveau JHJ. Variation in local carrying capacity and the individual fate of bacterial colonizers in the phyllosphere. ISME J. 2012;6:756-65.

45. Remus-Emsermann MNP, de Oliveira S, Schreiber L, Leveau JHJ. Quantification of lateral heterogeneity in carbohydrate permeability of isolated plant leaf cuticles. Front Microbiol. 2011;2:197.

46. Overbeek R, Olson R, Pusch GD, Olsen GJ, Davis JJ, Disz T, Edwards RA, Gerdes S, Parrello B, Shukla M, et al. The SEED and the Rapid Annotation of microbial genomes using Subsystems Technology (RAST). Nucleic Acids Res. 2014;42: D206-14.

47. Joyner DC, Lindow SE. Heterogeneity of iron bioavailability on plants assessed with a whole-cell GFP-based bacterial biosensor. Microbiology-Uk. 2000;146: 2435-45.

48. Morgan J, Tukey Jr H. Characterization of leachate from plant foliage. Plant Physiol. 1964;39:590.

49. Tukey Jr HB. Leaching of metabolites from above-ground plant parts and its implications. Bull Torrey Bot Club. 1966;93:385-401.

50. Aziz RK, Bartels D, Best AA, DeJongh M, Disz T, Edwards RA, Formsma K, Gerdes S, Glass EM, Kubal M, et al. The RAST Server: Rapid Annotations using Subsystems Technology. BMC Genomics. 2008;9:1-15.

51. Devoid S, Overbeek R, DeJongh M, Vonstein V, Best AA, Henry C. Automated genome annotation and metabolic model reconstruction in the SEED and Model SEED. Methods Mol Biol. 2013;985:17-45. doi:10.1007/978-1 62703-299-5 2.

52. Henry CS, DeJongh M, Best AA, Frybarger PM, Linsay B, Stevens RL. Highthroughput generation, optimization and analysis of genome-scale metabolic models. Nat Biotechnol. 2010;28:977-82.

53. Tukey $\mathrm{Jr} \mathrm{H}$, Tukey $\mathrm{Sr} \mathrm{H}$. The loss of organic and inorganic materials by leaching from leaves and other above-ground plant parts. In: Radioisotopes in plant-soil nutrition studies. 1962. p. 289-302.

54. Liu H, Xu J, Liang R, Liu J. Characterization of the Medium- and Long-Chain n-Alkanes Degrading Pseudomonas aeruginosa Strain SJTD-1 and Its Alkane Hydroxylase Genes. PLOS ONE. 2014;9:e105506.

55. Throne-Holst M, Wentzel A, Ellingsen TE, Kotlar H-K, Zotchev SB. Identification of novel genes involved in long-chain n-alkane degradation by Acinetobacter sp. strain DSM 17874. Appl Environ Microbiol. 2007;73:3327-32. 
56. Freimoser FM, Pelludat C, Remus-Emsermann MNP. Tritagonist as a new term for uncharacterised microorganisms in environmental systems. ISME J. 2015;10(1):1-3.

57. Patrick J, Steains K. Auxin-promoted transport of metabolites in stems of Phaseolus vulgaris L:: auxin dose-response curves and effects of inhibitors of polar auxin transport. J Exp Bot. 1987;38:203-10.

58. Bhatia M, Girdhar A, Tiwari A, Nayarisseri A. Implications of a novel Pseudomonas species on low density polyethylene biodegradation: an in vitro to in silico approach. SpringerPlus. 2014:3:497.

59. Miyazaki R, Bertelli C, Benaglio P, Canton J, De Coi N, Gharib WH, Gjoksi B, Goesmann A, Greub G, Harshman K, et al. Comparative genome analysis of Pseudomonas knackmussii B13, the first bacterium known to degrade chloroaromatic compounds. Environ Microbiol. 2015;17:91-104.

60. Sandhu A, Halverson $\sqcup$, Beattie GA. Bacterial degradation of airborne phenol in the phyllosphere. Environ Microbiol. 2007;9:383-92.

61. Scheublin TR, Leveau JH. Isolation of Arthrobacter species from the phyllosphere and demonstration of their epiphytic fitness. Microbiologyopen. 2013;2:205-13.

62. Woese CR, Kandler O, Wheelis ML. Towards a natural system of organisms: proposal for the domains Archaea, Bacteria, and Eucarya. Proc Natl Acad Sci U S A. 1990;87:4576-9.

63. Garrity GM, Bell JA, Lilburn T. Phylum XIV. Proteobacteria phyl. nov. In: Garrity GM, Brenner DJ, Krieg NR, Staley JT, editors. Bergey's Manual of Systematic Bacteriology. 2nd Ed. Volume 2, Part B. New York: Springer; 2005. p. 1.

64. Garrity GM, Bell JA, Lilburn T. Class III. Gammaproteobacteria class. nov. In: Garrity GM, Brenner DJ, Krieg NR, Staley JT, editors, Bergey's Manual of Systematic Bacteriology, 2nd Ed. Volume 2, Part B. New York: Springer; 2005. p. 1.

65. Orla-Jensen S. The Main Lines of the Natural Bacterial System. J Bacteriol. 1921;6:263-73.

66. Skerman VBD, McGowan V, Sneath PHA. Approved lists of bacterial names. Int J Syst Evol Microbiol. 1980;30:225-420.

67. Winslow CEA, Broadhurst J, Buchanan RE, Krumwiede C, Rogers LA, Smith GH. The Families and Genera of the Bacteria: Preliminary Report of the Committee of the Society of American Bacteriologists on Characterization and Classification of Bacterial Types. J Bacteriol. 1917;2:505-66.

68. Migula W. Über ein neues System der Bakterien. In: Arbeiten aus dem Bakteriologischen Institut der Technischen Hochschule zu Karlsruhe. 1894. p. 235-8.

69. Ashburner M, Ball CA, Blake JA, Botstein D, Butler H, Cherry JM, Davis AP, Dolinski K, Dwight SS, Eppig JT, et al. Gene Ontology: tool for the unification of biology. Nat Genet. 2000;25:25-9.

70. Stothard P, Wishart DS. Circular genome visualization and exploration using CGView. Bioinformatics. 2005;21:537-9.

\section{Submit your next manuscript to BioMed Central and we will help you at every step:}

- We accept pre-submission inquiries

- Our selector tool helps you to find the most relevant journal

- We provide round the clock customer support

- Convenient online submission

- Thorough peer review

- Inclusion in PubMed and all major indexing services

- Maximum visibility for your research

Submit your manuscript at www.biomedcentral.com/submit

) Biomed Central 